\title{
Can rumen bacteria communicate to each other?
}

\author{
Mi-Young Won ${ }^{1}$, Linda B. Oyama², Stephen J. Courtney ${ }^{2}$, Christopher J. Creevey ${ }^{2}$ and Sharon A. Huws ${ }^{2^{*}}$ (D)
}

\begin{abstract}
Background: The rumen contains a myriad of microbes whose primary role is to degrade and ferment dietary nutrients, which then provide the host with energy and nutrients. Rumen microbes commonly attach to ingested plant materials and form biofilms for effective plant degradation. Quorum sensing (QS) is a well-recognised form of bacterial communication in most biofilm communities, with homoserine lactone (AHL)-based QS commonly being used by Gram-negative bacteria alone and Al-2 Lux-based QS communication being used to communicate across Gram-negative and Gram-positive bacteria. However, bacterial cell to cell communication in the rumen is poorly understood. In this study, rumen bacterial genomes from the Hungate collection and Genbank were prospected for QS-related genes. To check that the discovered QS genes are actually expressed in the rumen, we investigated expression levels in rumen metatranscriptome datasets.
\end{abstract}

Results: A total of 448 rumen bacterial genomes from the Hungate collection and Genbank, comprised of 311 Gram-positive, 136 Gram-negative and 1 Gram stain variable bacterium, were analysed. Abundance and distribution of AHL and Al-2 signalling genes showed that only one species (Citrobacter sp. NLAE-zl-C269) of a Gram-negative bacteria appeared to possess an AHL synthase gene, while the Lux-based genes (Al-2 QS) were identified in both Gram-positive and Gram-positive bacteria (191 genomes representing 38.2\% of total genomes). Of these 192 genomes, 139 are from Gram-positive bactreetteria and 53 from Gram-negative bacteria. We also found that the genera Butyrivibrio, Prevotella, Ruminococcus and Pseudobutyrivibrio, which are well known as the most abundant bacterial genera in the rumen, possessed the most lux-based Al-2 QS genes. Gene expression levels within the metatranscriptome dataset showed that Prevotella, in particular, expressed high levels of LuxS synthase suggesting that this genus plays an important role in QS within the rumen.

Conclusion: This is the most comprehensive study of QS in the rumen microbiome to date. This study shows that Al-2-based QS is rife in the rumen. These results allow a greater understanding on plant-microbe interactions in the rumen.

Keywords: Rumen, Quorum sensing, Bacteria, Acyl-homoserine lactone, LuxS, Al-2

\section{Background}

The rumen microbiome is a highly specialised, complex community composed of bacteria, protozoa, fungi and viruses [1]. Even though the rumen microbiome has been studied for many years, progress in understanding rumen microbial function has been slow due to the complexity of the ecosystem and available technologies, although recent 'omic advances have

\footnotetext{
* Correspondence: S.Huws@qub.ac.uk

${ }^{2}$ School of Biological Sciences, Institute for Global Food Security, Queen's University of Belfast, 19 Chlorine Gardens, Belfast BT9 5DL, Northern Ireland, UK

Full list of author information is available at the end of the article
}

improved our understanding. Despite these recent developments of 'omic technologies, there remains to be a dearth of knowledge regarding bacterial cell to cell communication in the rumen microbiome. Much is known about cell communication from pure culture studies of non-ruminant origin (e.g. Vibrio harveyi, $V$. fischeri, Escherichia coli, Pseudomonas aeruginosa), which shows that Gram-negative bacteria typically use acylated homoserine lactone (AHLs)-based quorum sensing (QS) cell to cell communication strategies (autoinducer-1 QS system; AI-1) and Gram-positive bacteria use furanosyl borate diester or tetrahydroxy furan (autoinducer-2 QS system; AI-2) [2-4]. In these

(c) The Author(s). 2020 Open Access This article is distributed under the terms of the Creative Commons Attribution 4.0 International License (http://creativecommons.org/licenses/by/4.0/), which permits unrestricted use, distribution, and 
bacteria, upregulation of QS autoinducer chemicals, followed by receptor binding, instigates changes in overall bacterial gene expression and phenotype, with increased virulence commonly being a consequence [4]. These studies outline the genetic basis of QSbased bacterial cell to cell communication in vitro and are useful for furthering our understanding; however, their applicability to microbiome communities are uncertain and cell to cell communication in multispecies biofilms, such as those in the rumen, are likely to be far more complicated.

AHLs have been detected in rumen fluid, suggesting they may play a role in cell to cell communication in the rumen microbiome [5]. By testing pure cultures, Erikson et al. (2002) [5] were not able to identify which bacteria produce AHL using Gram-negative rumen bacterial pure cultures, including Anaerovibrio lipolyticus 5S, Fibrobacter succinogenes S85, Megasphaera elsdenii LC1, Prevotella albensis 223/M2/7, P. brevis GA33, P. bryantii B14, P. ruminocola 23 and B85, Ruminobacter amylophilus 70 and WP109, Selenomonas ruminantium HD4, four unnamed S. ruminantium strains and Succinovibrio dextrinosolvens 24, as well as Gram-positive ruminal pure culture bacteria Butyrivibrio fibrisolvens, Lachnospira multiparus 20, Ruminococcus albus B199, two strains of R. flavefaciens and Streptococcus bovis YM150. This suggests that the as yet unculturable bacteria or other cultured bacteria not tested by Erickson et al. (2002) [5] may be responsible for most of the AHL signals found in rumen fluid. Interestingly, it has been reported that AHL signals are reduced in concentration in rumen fluid taken during the winter, possibly due to changes in the microbiome in line with winter diets [6]. While recently, Yang and colleagues isolated $P$. aeruginosa $\mathrm{YZ}_{1}$ from the rumen of cattle, which utilises AHL signals to communicate [7], although this bacterial genus is not commonly found in the rumen ecosystem [8]. Supporting the importance of cell to cell communication in the rumen microbiome, LuxS proteins and analogues from the AI-2 QS system have been annotated in transcriptome datasets from the rumen [9]. Moreover, AI-2 activity was also detected in the rumen contents of three cows [1012]. Mitsumori and colleagues (2003) [10] also detected AI-2 signals in pure cultures of B. fibrisolvens, Eubacterium ruminantium, Ruminococcus flavefaciens and Succinomonas amylolytica, suggesting a prominent role of AI-2-based QS in the rumen.

Recently, the Hungate collection of rumen microbial genomes was released [13]. This collection of 501 microbial genomes is the most comprehensive for the rumen microbiome and represents a major step change in our ability to understand the rumen microbiome. In this study, we took advantage of the availability of the Hungate collection, alongside other rumen bacterial genomes deposited in Genbank, to prospect rumen bacterial genomes for genes/proteins which are known to be involved in QS cell to cell communication and to further our understanding of the importance of QS in the rumen. We also confirmed that putative QS genes were expressed by prospecting for these sequences in the largest available rumen metatranscriptome datasets.

\section{Methods}

\section{Bacterial genomes used in this study}

The bacterial genomes used in this study were obtained both from the Hungate collection (428 bacterial species excluding genomes from archaea, viruses and eukaryotes) (https://genome.jgi.doe.gov/portal/HungateCollection/ HungateColl-ection.info.html) (accessed on July 2018) and from Genbank (https://www.ncbi.nlm.nih.gov/genome) (July 2018) [13]. To find the genomes in Genbank, the genomes category in PubMed was searched using the keywords "rumen and bacteria". Only Genbank results from complete genomes not represented in the Hungate collection were used in this study. The Genbank search produced 20 rumen bacteria genomes, 12 Gram-positive and 8 Gram-negative species. Combined with the 428 bacterial genomes from the Hungate collection, this resulted in genomes from a total of 448 rumen bacteria species, 311 which were Gram-positive, 136 which were Gramnegative and 1 which was Gram stain variable (Additional file 1: Table S1). These were then analysed for genes involved in QS cell to cell communication.

\section{Prospecting genomes for quorum sensing-related proteins}

Each of the genomes was re-annotated using PROKKA (using default settings), which uses BLAST + and Blastp to annotate, and for each genome, the resulting annotations of the predicted protein sequences were searched using semantic approaches [14]. To search for AHLrelated genes, the following search terms were prospected: Quorum sensing, autoinducer, AHL, HSL, homoserine lactone, $\mathrm{N}$-acyl homoserine, AHL synthase, RhII, LuxI, LuxR, LasI, LasR and homoserine lactone efflux protein. To search for AI-2-related genes, the following search terms were prospected: Quorum sensing, autoinducer, LuxS, LuxP, LuxQ, S-ribosylhomocysteine, S-ribosylhomocysteine lyase (full chemical name for Lux S) and AI-2.

\section{Sequence alignment and generation of phylogenetic trees}

All LuxS proteins identified from the rumen bacteria were aligned using Mega7 (v. 7.0.26) [15]. A phylogenetic tree was constructed with additional protein genes from Vibrio harveyi (Gram-negative) and Streptococcus pneumoniae (Gram-positive), in which LuxS based on QS system have 
been well studied, alongside LuxS-based QS proteins previously identified from rumen microbial metagenomics and metatranscriptomic sequences [9], for comparative purposes. Phylogenetic trees were constructed using neighbour-joining clustering method [16]. A bootstrap consensus tree was inferred from 1000 replicates, and branches corresponding to partitions reproduced in less than $50 \%$ bootstrap replicates are collapsed. The evolutionary distances were computed using the Poisson correction method and are represented by in number of amino acid substitutions per site. The analysis involved 201 amino acid sequences. All ambiguous positions were removed for each sequence pair. There were a total of 191 positions in the final dataset.

\section{LuxS synthase expression within a rumen bacterial metatranscriptome}

Twenty publicly available metatranscriptomic datasets were taken from the National Center for Biotechnology Information Sequence Read Archive, under the accession number SRA075938 [16]. The samples were $150 \mathrm{bp}$ paired-end reads from the Illumina Hiseq2000 sequencer [16]. Fastq files were processed with multiqc [17], and reads were trimmed from 150 to $110 \mathrm{bp}$ using the Trimmomatic software version 0.36 [18]. Reads were aligned to the Hungate rumen genome dataset using bowtie2 version 2.3.0 [19] using the settings "--very-sensitive-local" which allowed soft trimming of the reads and a relaxed alignment and "-k 497". The resulting SAM files were converted to BAM files using SAMtools version 1.9 [20]. SAMtools version 1.9 was used to filter all and the best alignment position for each read using the flag option "-F 260 ". For each of the 20 final filtered BAM files FeatureCounts (from the subread package version 2.0.0) [21] was used to calculate the number of reads that align within the boundaries of every predicted gene in the Hungate genomes. The read counts were then converted into RPKM values. Finally, the RPKM values of the genes of interest in this study were extracted from the entire expression count table.

\section{Results}

\section{Quorum sensing-related proteins with rumen bacterial genomes}

For the purpose of analysis, two genera, Lachnobacterium and Micrococcus, which are often described as weakly Gram-positive or Gram-variable respectively were included with the Gram-positive bacteria group (Additional file 1: Table S1). Flavonifractor plautii, which is also a Gram-variable bacterium, but never referred to as being weakly Gram-positive or negative, was consequently not included in either group (Additional file 1: Table S1). Fifty percent of the genome sequences prospected for QS cell to cell signalling did not contain any protein annotated with LuxS or AHL signalling system, irrespective of their Gram staining phenotypes. Thus, any genomes which did not have LuxS or AHL signalling genes were presented as N/A (not applicable; Additional file 1: Table S1). When specific species names or strain ID could not be identified from Genbank genome entries, results were included with closely related genera or families in the Hungate collection (e.g. family: Crodiobacteriaceae, genus: Olsenella and family: Propionibacteriaceae, genus: Propionibacterium). This was also done when species had been re-classified with different names, e.g. Clostridium mangenotii and Eubacterium rectalis have been re-classified as Clostridioides mangenotii and Agathobacter rectalis respectively.

LuxS proteins, which can produce/regulate AI-2 signal and the corresponding receptor protein LuxR, were highly represented in both Gram-positive and Gram-negative bacteria species. A total of 171 LuxS proteins were identified in the genomes analysed, with $40.2 \%$ and $33.0 \%$ of LuxS proteins being found within Gram-positive and Gram-negative bacteria species respectively (Fig. 1; Additional file 1: Table S1). A total of 21 LuxR proteins were also identified in the genomes analysed, with $4.5 \%$ and $5.1 \%$ of LuxR proteins being found within Gram-positive and Gram-negative bacteria species respectively (Fig. 1; Additional file 1: Table S1). In contrast, only one species (Citrobacter sp. NLAE-zl-C269) appeared to have the AHL synthase gene among the Gram-negative bacteria list (Additional file 1: Table S1). Nonetheless, AHL efflux proteins were detected in six (Acinetobacter, Enterobacter, Porphyromonadaceae, Prevotella, Oxalobacter, Wolinella) Gram-negative bacterial genomes (4.4\%), with no AHL-related proteins being detected in Gram-positive bacteria as expected. Citrobacter sp. NLAE-zl-C269 also possessed luxS and LuxR genes, suggesting that this bacterium may use the AHL and AI-2 quorum sensing systems (Fig. 1; Additional file 1: Table S1). On further note, nine of the rumen bacterial genomes (Bacillus sp. MB2021, Blautia schinkii DSM 10518, Dorea longicatena AGR2136, Lachnospiraceae bacterium FE2018, Slackia heliotrinireducens, Oscillibacter sp. PC13, Oxalobacter formigenes, Sagittula stellata, Selenemonas ruminantium) also contained AHL lactonase genes which are used to cleave the lactone ring. This suggests that these rumen bacteria may have the ability to reduce AHL-based QS in the rumen through degradation of AHL.

As earlier stated, the LuxS protein was detected in $40.2 \%$ (126 genomes) and 33.0\% (45 genomes) of Grampositive and Gram-negative species respectively, contained within a total of 1711 different genera. Among them, the largest number of LuxS protein genes was 


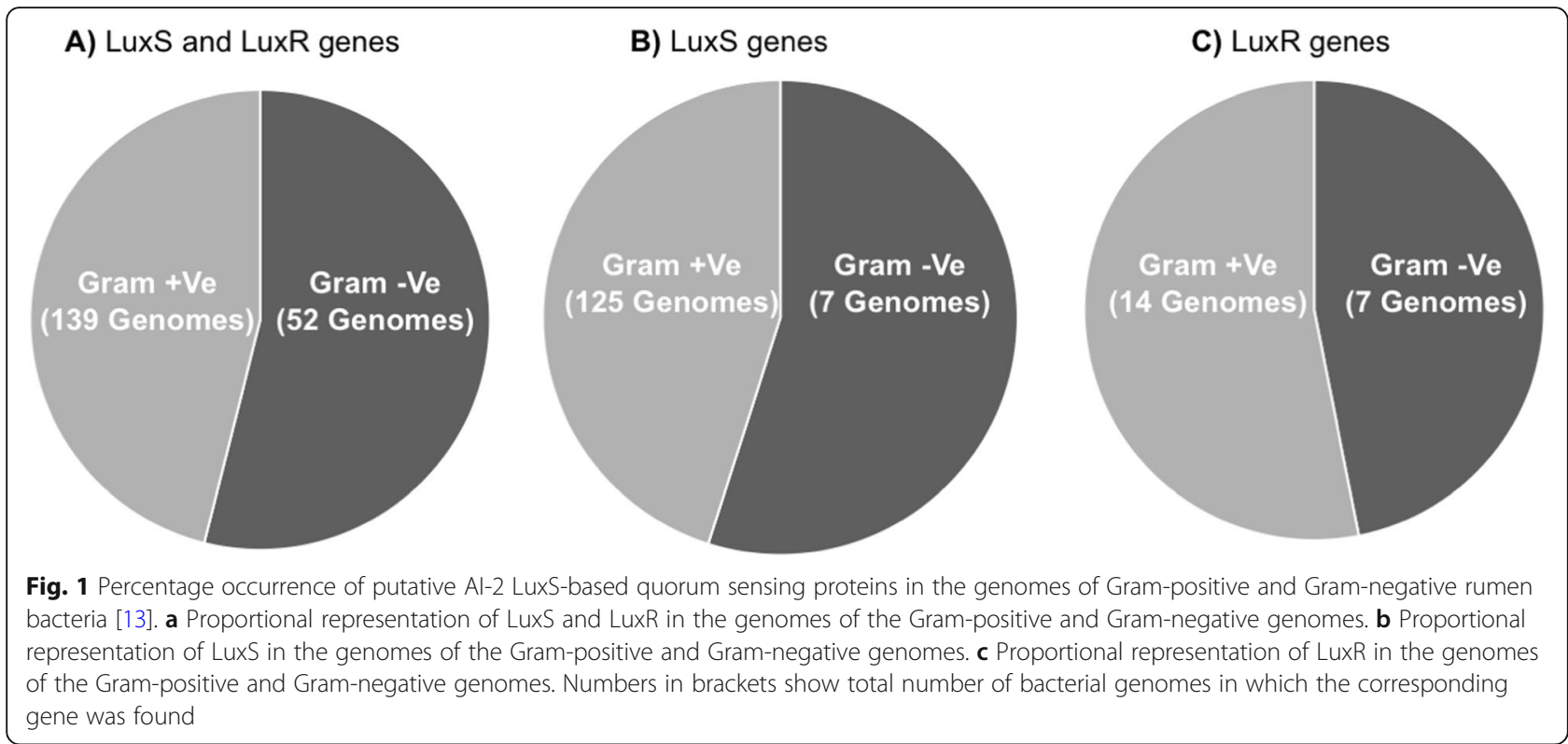

detected with the genus Butyrivibrio (48 species), followed by Pseudobutyrivibrio (15 species), Prevotella (14 species) and Ruminococci (10 species) (Fig. 2). Furthermore, a phylogenetic tree including the LuxS protein sequences from the 171 putatively positive LuxS species was generated to visualise their similarity. Vibrio harveyi and Streptococcus pneumoniae LuxS proteins, alongside LuxS proteins identified by Ghali et al. (2016) [9], were used for comparison (Fig. 3). From this phylogenetic tree, it can be observed that LuxS proteins from the same rumen bacterial genera generally clustered together and showed high similarity to each other compared with Vibrio harveyi and Streptococcus pneumoniae, although multiple clustering of LuxS proteins can occur within the same genera. For instance, LuxS proteins from the Butyrivibrio genus formed at least 8 phylogenetically distinct clusters (Fig. 4). Also of note is the fact that LuxS proteins previously encountered in rumen metagenomics and metatranscriptomic datasets [9] are different to those found within the genomes of cultured bacteria

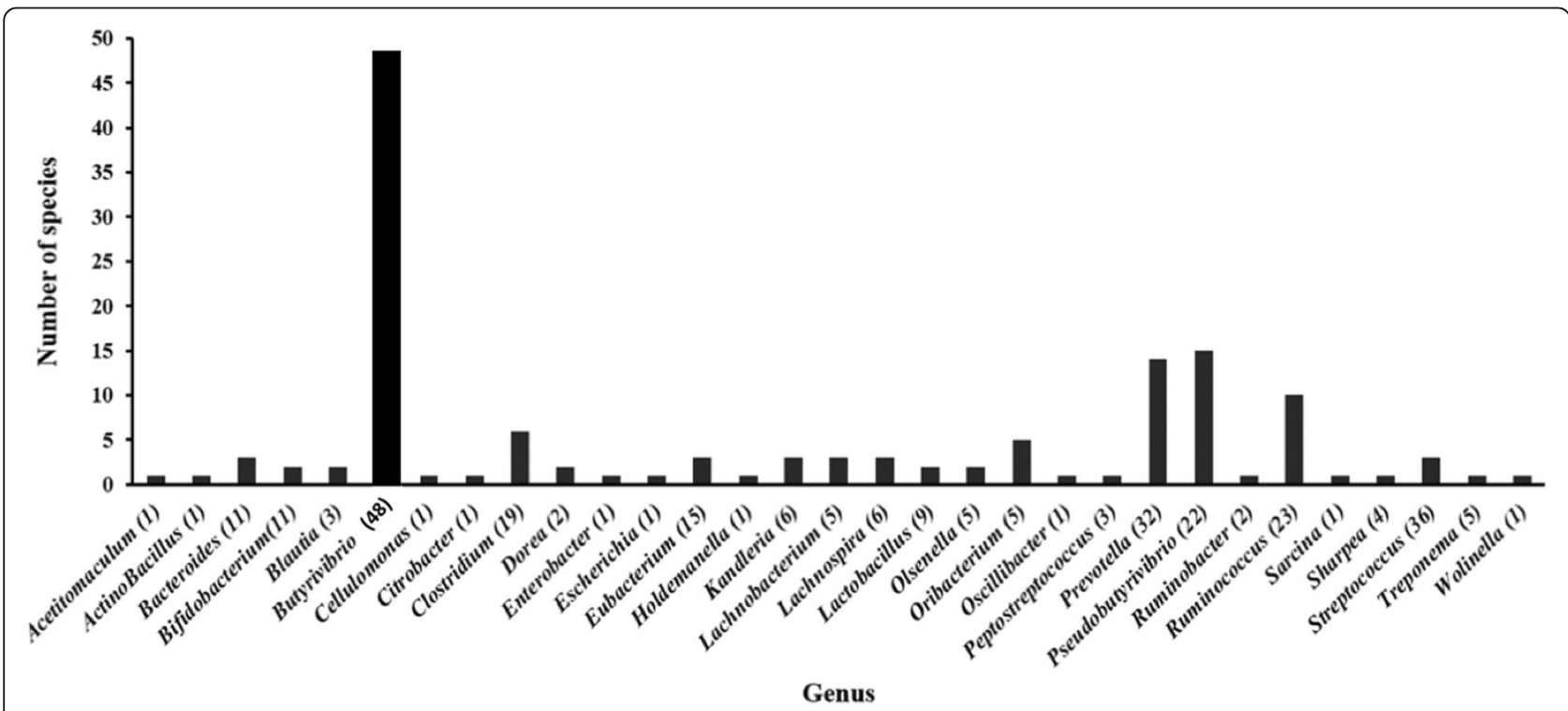

Fig. 2 Distribution and abundance of LuxS proteins relating to Al-2-based quorum sensing across 448 rumen bacterial genomes [13] 


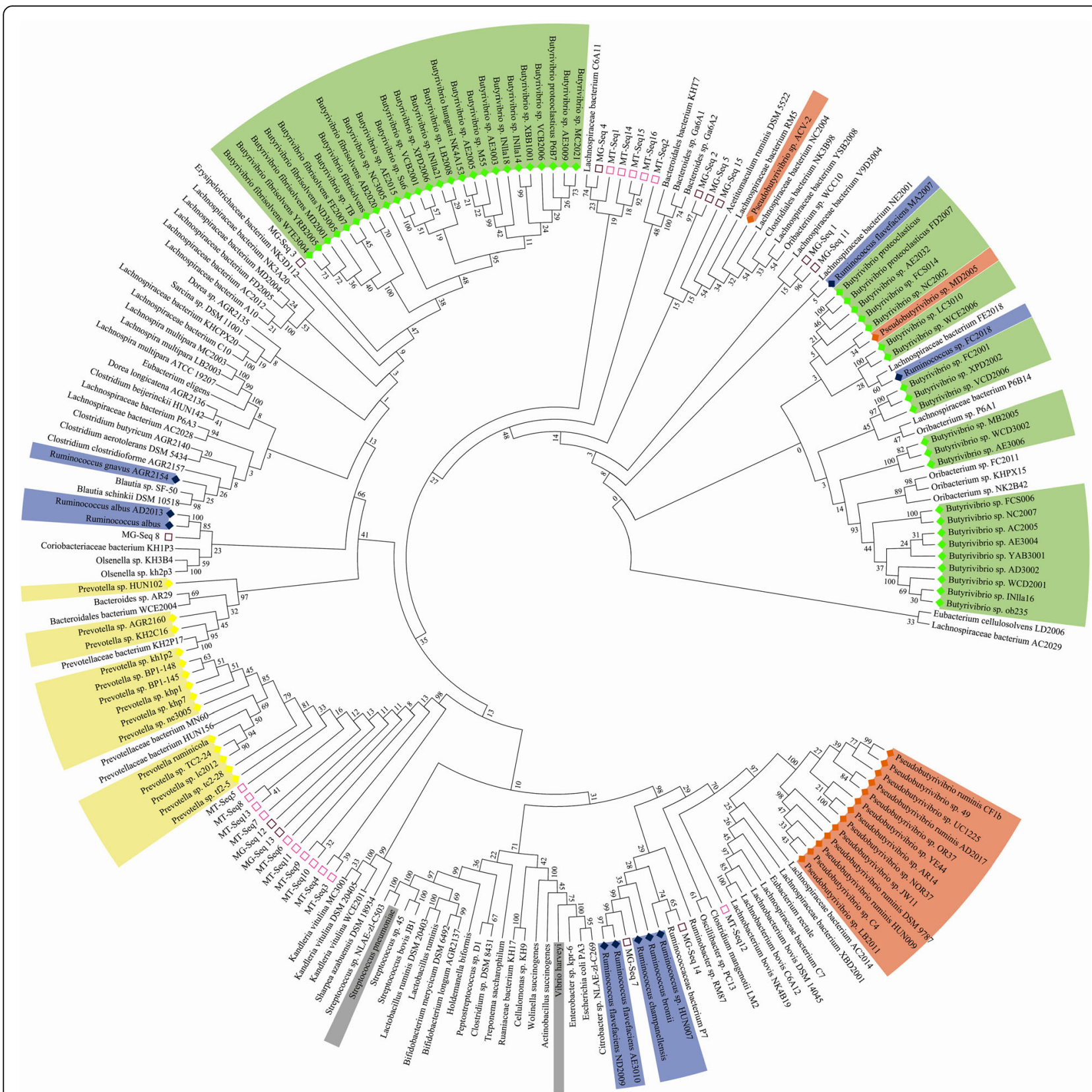

Fig. 3 Comparative phylogenetic tree of all putative LuxS proteins detected within the genomes of 171 rumen bacterial species. Two standards (Vibrio harveyi and Streptococcus pneumoniae) were used for comparison with other samples (highlighted with grey colour). Most abundant four groups were highlighted with colours (orange: Pseudobutyrivibrio, yellow: Prevotella, green: Butyrivibrio and blue: Ruminococcus). Sequences denoted by a square and beginning with MG are those identified by Ghali et al. [9] from rumen metagenomic sequences and those with MT also being derived from the same study using a metatranscriptome dataset

used in this study (Fig. 4), indicating that the LuxS proteins have numerous haplotypes in the rumen, perhaps indicative of the importance of this communication system in this ecosystem. It should also be noted that the LuxS protein sequences investigated within Ghali et al.'s study [9] clustered similarily to each other into two separate clades within this study in line with the phylogenetic trees published by the authors. Our study adds significantly more information to this initial publication due to the recent increased availability of rumen bacterial genomes [13].

\section{Expression of the identified LuxS synthase genes in rumen metatranscriptome datasets}

In order to investigate whether the identified LuxS genes within the hungate genomes [16] are actually expressed 


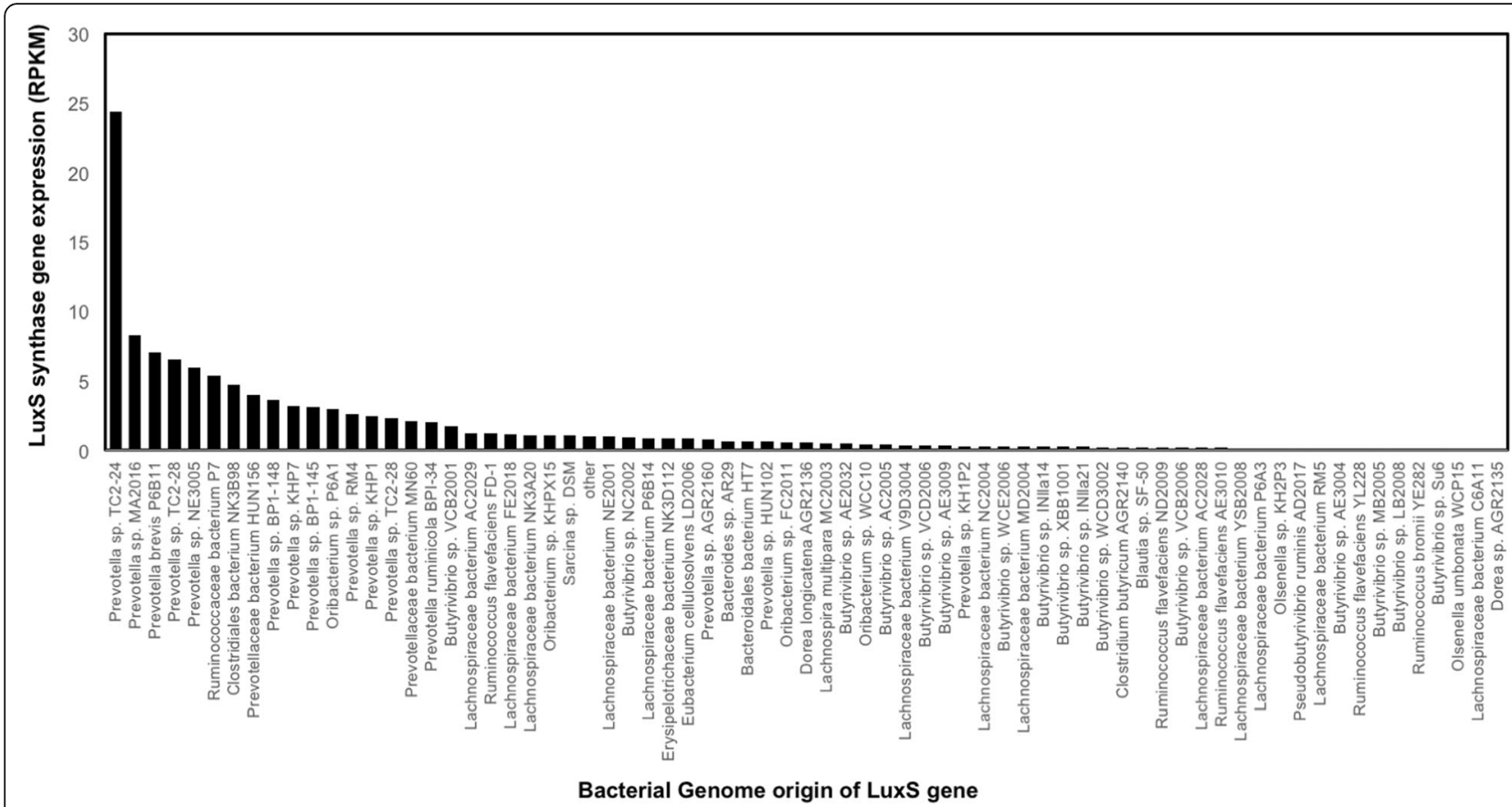

Fig. 4 Average expression of LuxS genes identified in the bacterial genomes within rumen bacterial metatranscriptome datasets [16]. Expression is shown as reads per kilobase of transcript per million (RKPM). Where RKPM was $<0.1$, the expression data was grouped as "other". Other essentially contained the expression of the LuxS synthase genes discovered within Lachnobacterium bovis DSM, Ruminococcus gnavus AGR2154, Actinobacillus succinogenes 130Z, Streptococcus sp. NLAE-zl-C503, Butyrivibrio sp. AD3002, Butyrivibrio sp. INIla18, Pseudobutyrivibrio ruminis DSM, Bifidobacterium bifidum Calf96, Butyrivibrio sp. TB, Butyrivibrio sp. WCD2001, Succinimonas_amylolytica DSM 2873, Pseudobutyrivibrio sp. JW11, Ruminococcus flavefaciens 17, Pseudobutyrivibrio sp. LB2011, Ruminococcus albus SY3, Pseudobutyrivibrio sp. MD2005, Butyrivibrio sp. NC3005, Clostridium mangenotii LM2, Cellulomonas sp. KH9, Clostridium aerotolerans DSM, Butyrivibrio fibrisolvens YRB2005, Butyrivibrio fibrisolvens MD2001, Kandleria vitulina WCE2011 and Butyrivibrio sp. YAB300

in the rumen, we investigated their expression within one of the largest rumen metatranscriptome datasets available [16] (105 GB sequencing data). We found expression of 97 (out of 171, 56.7\% of the LuxS genes within these datasets (Fig. 4). In order to evaluate expression, we took the average expression across all 20 of the metratranscriptome sequences from the Shi et al. [16] study. On average, Prevotella spp. expressed the most LuxS, particularly Prevotella sp. TC2-24 (Fig. 4). High expression levels were also seen for LuxS genes originally detected within Ruminococcaceae bacterium P7, Clostridiales bacterium NK3B98, Prevotellaceae bacterium HUN156 and MN60, Oribacterium sp. P6A1 and Butyrivibrio sp. VCB2001 (Fig. 4). It should, of course, be noted that the expression data will be proportional to the abundance of the bacteria within the metranscriptome datasets and as such they show a snap shot in time, under those particular circumstances, but expression may differ under altering conditions. It also means that whilst we did not see the expression of 74 of the originally identified LuxS genes within the metatranscriptome datasets, it does not mean that they categorically are not expressed and may well be found under different ecological circumstances. The purpose of investigating the expression of the LuxS genes identified within the Hungate collection was to show that most are expressed and therefore are likely used for QS in the rumen.

\section{Discussion}

Bacterial quorum sensing is known to have major implications for bacterial phenotype in pure culture-based studies [22-24]. However, quorum sensing mechanisms in multi-species biofilm communities are poorly understood due to the complexities of these microbiomes. In this study, we used rumen bacterial genomes from the Hungate collection, a large database which is largely representative of many of the rumen microorganisms (501 bacterial and archaeal genomes) [13] and complete rumen bacterial genomes from Genbank to prospect for the abundance and distribution of AHL and AI-2-based QS systems. For ease of analysis and data visualisation, the bacteria genomes were categorised according to taxonomic information as well as their Gram staining phenotypes. From a total of 448 bacteria species recorded in the Hungate collection (at the time data was accessed for analysis) and Genbank genomes, 311 
species were Gram-positive bacteria and 136 were Gram-positive representing $69.4 \%$ and $30.6 \%$ of the bacteria used within this study.

We found only one species (Citrobacter sp. NLAE-zlC269) out of 136 bacterial genomes from Gram-negative bacteria analysed possessed an AHL synthase gene. Citrobacter spp. have been known to have the ability to hydrolyse cellulose in the rumen and have been reported in non-rumen-based studies to produce 3-hydroxyl type of AHLs $[25,26]$. We did, however, also find evidence that the rumen Citrobacter spp. genome also possessed AI-2 QS capacity, a phenomenon which to our knowledge has not been shown before in other Citrobacter sp.

Approximately $40.2 \%$ and $33.0 \%$ of LuxS genes were identified from Gram-positive and Gram-negative bacterial species respectively. Therefore, our results also confirm that the LuxS proteins may be utilised by both Gram-positive and negative bacteria as previously suggested [27-29]. Approximately $40 \%$ of the studied rumen bacterial genomes contained LuxS genes, with these predominating in Gram-positive bacteria (40.5\% from the 311 Gram-positive bacterial genomes and 33.1\% from the 136 Gram-negative bacterial genomes). Sequence alignment and visualisation using a phylogenetic tree show that the LuxS genes found in the rumen bacterial genomes were highly similar to each other compared with Vibrio harveyi or Streptococcus pneumoniae LuxS genes, indicating that LuxS genes in the rumen may be highly conserved within each genus but are also distantly related to non-ruminal LuxS genes. Also, noteworthy was the fact that the QS genes found within this study were different to those found by Ghali et al. (2016) [9], which may be consequences of the large number of genomes prospected in this study. Irrespectively, the presence of numerous LuxS haplotypes suggests that this QS system may be especially important for bacterial communication within the rumen.

Overall, we show evidence that Butyrivibrio, Prevotella, Ruminococcus and Pseudobutyrivibrio rumen bacterial genomes, which are concomitantly the most abundant bacterial genera in the rumen have the capacity to use AI-2-based quorum sensing. This data also suggests that quorum sensing is ubiquitous within the rumen in general. This observation is in agreement with those from many recent studies, which show that these bacterial genera may possess AI-2 QS signalling ability [9-11]. Further observations from this study suggest that bacteria with LuxS protein predominate in the rumen, and it has become clear that the AI-2 signal has a great preponderance in the rumen. The expression results for the identified LuxS genes within large published metatranscriptome datasets [16] confirmed that 97 of the 171 identified LuxS genes were expressed in the rumen. This data also showed that rumen Prevotella likely play a major role in AI-2-based QS in the rumen. It has also been shown that oral Prevotella sp. possesses AI-2 QS capacity [30].

However, many ruminant bacteria are yet to be identified, cultured or genome sequenced, and further studies to identify them and their potential involvement in QS in the rumen are paramount. It may also be important to assess the contribution of other rumen organism types including fungi, archaea, protozoa and viruses to QS in the rumen and their role in plant degradation alongside bacteria given that several non-ruminal studies show that other microorganisms such as fungi, archaea and protozoa also use QS signalling [31-36]. Moreover, there are still a lot of Gram-negative bacteria that remain uncultured with the potential to utilise AHL-based QS in the rumen. Thus, it is important to improve our knowledge of both AI-2 and AHL QS signalling system in the rumen to better understand bacterial interactions and consequences on ruminant production. Further, in vitro analysis to confirm the bioluminescence activity of the LuxS genes detected in these rumen bacteria genera will be required to understand their expression and role in the rumen microbiome.

\section{Conclusion}

Using the major genomic resources available, we show on a greater scale than previously possible that QS is likely to be an important phenomenon in the rumen microbiome. Our data suggest that AI-2-based QS is probably the most abundant and perhaps most important signal used by rumen bacteria. Further research into the implications of rumen-based QS on plant degradation and nutrient availability for the host is now required.

\section{Supplementary information}

Supplementary information accompanies this paper at https://doi.org/10. 1186/s40168-020-00796-y.

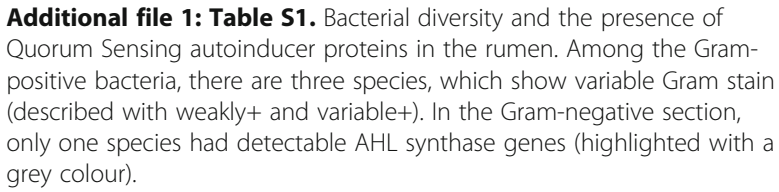
Quorum Sensing autoinducer proteins in the rumen. Among the Grampositive bacteria, there are three species, which show variable Gram stain (described with weakly+ and variable+). In the Gram-negative section, only one species had detectable AHL synthase genes (highlighted with a grey colour)

Authors' contributions

MY, SH and LO designed the study and CC and SC advised reagrding computational analysis.All authors read and approved the final manuscript.

Competing interests

The authors declare that they have no competing interests.

Author details

${ }^{1}$ Institute of Biological, Environmental and Rural Sciences (IBERS), Aberystwyth University, Penglais Campus, Aberystwyth SY23 3DA, UK. ${ }^{2}$ School of Biological Sciences, Institute for Global Food Security, Queen's 
University of Belfast, 19 Chlorine Gardens, Belfast BT9 5DL, Northern Ireland, UK.

Received: 4 April 2019 Accepted: 5 February 2020

Published online: 21 February 2020

\section{References}

1. Huws SA, Creevey CJ, Oyama LB, Mizrahi I, Denman SE, Popova M, MuñozTamayo R, et al. Addressing global ruminant agricultural challenges through understanding the rumen microbiome: past, present, and future. Front Microbiol. 2018:9:1-84.

2. Waters CM, Bassler BL. QUORUM SENSING: cell-to-cell communication in bacteria. Ann Rev Cell Develop Biol. 2005;21(1):319-46.

3. Galloway WRJD, Hodgkinson JT, Bowden SD, Welch M, Spring DR. Quorum sensing in Gram-negative bacteria: small-molecule modulation of AHL and Al-2 quorum sensing pathways. Chem Rev. 2011;111(1):28-67.

4. Pereira CS, Thompson JA, Xavier KB. Al-2-mediated signalling in bacteria. FEMS Microbiol Rev. 2013;37(2):156-81.

5. Erickson DL, Nsereko VL, Morgavi DP, Selinger LB, Rode LM, Beauchemin KA. Evidence of quorum sensing in the rumen ecosystem: detection of $\mathrm{N}$-acyl homoserine lactone autoinducers in ruminal contents. Can J Microbiol. 2002;48(4):374-8.

6. Edrington TS, Farrow RL, Sperandio V, Hughes DT, Lawrence TE, Callaway TR, et al. Acyl-homoserine-lactone autoinducer in the gastrointesinal tract of feedlot cattle and correlation to season, E. Coli 0157:H7 prevalence, and diet. Curr Microbiol. 2009;58(3):227-32.

7. Yang Y, Zhou M, Hardwidge PR, Cui H, Zhu G. Isolation and characterization of $\mathrm{N}$-acyl homoserine lactone-producing bacteria from cattle rumen and swine intestines. Front Cell Infect Microbiol. 2018;8:55.

8. Henderson G, Cox F, Ganesh S, Jonker A, Young W, Abecia L, et al. Rumen microbial community composition varies with diet and host, but a core microbiome is found across a wide geographical range. Sci Rep. 2015;5: 14567. https://doi.org/10.1038/srep14567.

9. Ghali I, Shinkai T, Mitsumori M. Mining of luxS genes from rumen microbial consortia by metagenomic and metatranscriptomic approaches. Anim Sci J. 2016;87(5):666-73.

10. Mitsumori M, Xu L, Kajikawa H, Kurihara M, Tajima K, Hai J, Takenaka A. Possible quorum sensing in the rumen microbial community: detection of quorum-sensing signal molecules from rumen bacteria. FEMS Microbiol Lett. 2003;219(1):47-52.

11. Lukáš F, Gorenc G, Kopečný J. Detection of possible Al-2-mediated quorum sensing system in commensal intestinal bacteria. Folia Microbiologica. 2008; 53(3):221-4.

12. Ran T, Zhou C, Xu L, Geng M, An Z, Tang S, Wang M, et al. Initial detection of the quorum sensing autoinducer activity in the rumen of goats in vivo and in vitro. J Int Agri. 2016;15(10):2343-52.

13. Seshadri R, Leahy SC, Attwood GT, Teh KH, Lambie SC, Cookson AL, Eloe-Fadrosh EA, et al. Cultivation and sequencing of rumen microbiome members from the Hungate1000 Collection. Nat Biotechnol. 2018;36(4):59-367.

14. Seemann T. Prokka: rapid prokaryotic genome annotation. Bioinformatics. 2014;30(14):2068-9.

15. Kumar S, Stecher G, Tamura K. MEGA7: molecular evolutionary genetics analysis version 7.0 for bigger datasets. Mol Biol Evol. 2016;33(7):870-1874.

16. Shi W, Moon CD, Leahy SC, Kang D, Froula J, Kittelmann S, Fan C, Deutsch S, Gagic D, Seedorf H, Kelly WJ, Atua R, Sang C, Soni P, Li D, Pinares-Patiño CS, McEwan JC, Janssen PH, Chen F, Visel A, Wang Z, Attwood GT, Rubin EM. Methane yield phenotypes linked to differential gene expression in the sheep rumen microbiome. Genome Res. 2014;24(9):1517-25. https://doi.org/ 10.1101/gr.168245.113.

17. Ewels P, Magnusson M, Lundin S, Käller M. MultiQC: summarize analysis results for multiple tools and samples in a single report. Bioinformatics. 2016;32(19):3047-8.

18. Bolger AM, Lohse $\mathrm{M}$, Usadel B. Trimmomatic: a flexible trimmer for Illumina sequence data. Bioinformatics. 2014;30(15):2114-20.

19. Langmead B, Salzberg SL. Fast gapped-read alignment with Bowtie 2 . Nature Methods. 2012;9(4):357.

20. Li H, Handsaker B, Wysoker A, Fennell T, Ruan J, Homer N, Marth G, Abecasis $\mathrm{G}$, Durbin R. The sequence alignment/map format and SAMtools. Bioinformatics. 2009;25(16):2078-9.
21. Liao Y, Smyth GK, Shi W. featureCounts: an efficient general purpose program for assigning sequence reads to genomic features. Bioinformatics. 2013;30(7):923-30

22. Kimura M. A simple method for estimating evolutionary rate of base substitutions through comparative studies of nucleotide sequences. J Mol Evol. 1981;16. https://doi.org/10.1007/BF01731581.

23. Kleerebezem M, Quadri LEN, Kuipers OP, De Vos WM. Quorum sensing by peptide pheromones and two-component signal-transduction systems in Gram-positive bacteria. Mol Microbiol. 1997;24(5):895-904.

24. Huang JJ, Han J, Zhang L, Leadbetter JR. Utilization of acyl-homoserine lactone quorum signals for growth by a soil Pseudomonad and Pseudomonas aeruginosa PAO1. Appl Environ Microbiol. 2003;69(10):5941-9.

25. Parsek MR, Greenberg EP. Sociomicrobiology: the connections between quorum sensing and biofilms. Trends Microbiol. 2005;13(1):27-33.

26. Coulthrus SJ, Clare S, Evans TJ, Foulds IJ, Roberts KJ, Welch M, et al. Quorum sensing has an unexpected role in virulence in the model pathogen Citrobacter rodentium. EMBO Reports. 2007;8(7):698-703.

27. Kher HL, Krishnan T, Letchumanan V, Hong K-W, How K-Y, Lee L-H, Tee K-K, et al. Characterization of quorum sensing genes and $\mathrm{N}$-acyl homoserine lactones in Citrobacter amalonaticus strain YG6. Gene. 2019;684:58-69.

28. De Keersmaecker SCJ, Vanderleyden J. Constraints on detection of autoinducer-2 (Al-2) signalling molecules using Vibrio harveyi as a reporter. Microbiol. 2003;149:1953-6.

29. Xavier KB, Bassler BL. LuxS quorum sensing: more than just a numbers game. Curr Opinion Microbiol. 2003;6(2):191-7.

30. Vendeville A, Winzer K, Heurlier K, Tang C.M, Hardie KR. Making "sense" of metabolism: autoinducer-2, LUXS and pathogenic bacteria. Nature Reviews Microbiol. 2005; 3(5), 383-396. doi:https://doi.org/10.1038/nrmicro1146

31. Henke JM, Bassler BL. Three parallel quorum-sensing systems regulate gene expression in Vibrio harveyi. J Bacteriol. 2014;186(20):6902-14.

32. Dixon EF, Hall RA. Noisy neighbourhoods: quorum sensing in fungalpolymicrobial infections. Cellular Microbiol. 2015;17(10):431-1441.

33. Rajput A, Kaur K, Kumar M. SigMol: repertoire of quorum sensing signaling molecules in prokaryotes. Nucleic Acids Res. 2015;44:D634-9.

34. Sun S, Tay QXM, Kjelleberg S, Rice SA, McDougald D. Quorum sensingregulated chitin metabolism provides grazing resistance to Vibrio cholerae biofilms. ISME J. 2015;9(8):1812.

35. Wongsuk T, Pumeesat $P$, Luplertlop N. Fungal quorum sensing molecules: role in fungal morphogenesis and pathogenicity. J Basic Microbiol. 2016; 56(5):440-7.

36. Charlesworth JC, Beloe C, Watters C, Burns BP. Quorum sensing in archaea: recent advances and emerging directions. Biocommun Archaea. 2017:11932.

\section{Publisher's Note}

Springer Nature remains neutral with regard to jurisdictional claims in published maps and institutional affiliations.

Ready to submit your research? Choose BMC and benefit from:

- fast, convenient online submission

- thorough peer review by experienced researchers in your field

- rapid publication on acceptance

- support for research data, including large and complex data types

- gold Open Access which fosters wider collaboration and increased citations

- maximum visibility for your research: over $100 \mathrm{M}$ website views per year

At $\mathrm{BMC}$, research is always in progress.

Learn more biomedcentral.com/submissions 